\title{
Developmental evolution of flowering plant pollen tube cell walls: callose synthase (CalS) gene expression patterns
}

\author{
Jason M Abercrombie, Brian C O'Meara, Andrew R Moffatt and Joseph H Williams*
}

\begin{abstract}
Background: A number of innovations underlie the origin of rapid reproductive cycles in angiosperms. A critical early step involved the modification of an ancestrally short and slow-growing pollen tube for faster and longer distance transport of sperm to egg. Associated with this shift are the predominantly callose (1,3- $\beta$-glucan) walls and septae (callose plugs) of angiosperm pollen tubes. Callose synthesis is mediated by callose synthase (CalS). Of 12 CalS gene family members in Arabidopsis, only one (CalS5) has been directly linked to pollen tube callose. CalS5 orthologues are present in several monocot and eudicot genomes, but little is known about the evolutionary origin of CalS5 or what its ancestral function may have been.

Results: We investigated expression of CalS in pollen and pollen tubes of selected non-flowering seed plants (gymnosperms) and angiosperms within lineages that diverged below the monocot/eudicot node. First, we determined the nearly full length coding sequence of a CalS5 orthologue from Cabomba caroliniana (CcCalS5) (Nymphaeales). Semi-quantitative RT-PCR demonstrated low CcCalS5 expression within several vegetative tissues, but strong expression in mature pollen. Cals transcripts were detected in pollen tubes of several species within Nymphaeales and Austrobaileyales, and comparative analyses with a phylogenetically diverse group of sequenced genomes indicated homology to CalS5. We also report in silico evidence of a putative CalS5 orthologue from Amborella. Among gymnosperms, CalS5 transcripts were recovered from germinating pollen of Gnetum and Ginkgo, but a novel Cals paralog was instead amplified from germinating pollen of Pinus taeda.

Conclusion: The finding that CalS5 is the predominant callose synthase in pollen tubes of both early-diverging and model system angiosperms is an indicator of the homology of their novel callosic pollen tube walls and callose plugs. The data suggest that CalS5 had transient expression and pollen-specific functions in early seed plants and was then recruited to novel expression patterns and functions within pollen tube walls in an ancestor of extant angiosperms.
\end{abstract}

\section{Background}

The pollen tube is a unique feature of male gametophytes of seed plants. In cycads and Ginkgo, pollen tubes are long-lived and function solely as haustorial, highly branched structures that grow invasively into female tissues [1-3]. In conifers and Gnetales pollen tubes function in a new way to deliver non-motile sperm to the egg (siphonogamy), while generally retaining a haustorial growth pattern [2,3]. Flowering plant (angiosperm) pollen tubes have lost most features of haustorial growth - their

\footnotetext{
* Correspondence: joewill@utk.edu

Department of Ecology and Evolutionary Biology, University of Tennessee, Knoxville, TN, USA
}

pollen tubes are typically short-lived and seem to function exclusively to deliver sperm to the egg $[4,5]$. The origin of siphonogamy has been held up as a classic example of exaptation [6], because the plesiomorphic function of the pollen tube - nutritional support for the male gametophyte - was subsequently co-opted for a novel role in sperm delivery [3]. Yet siphonogamy is clearly a complex process, and it is not at all obvious which aspects have common origins, which represent modifications of an ancestral pattern, and which have arisen independently in separate lineages $[1,3,4,7]$. Understanding the homologies of pollen tube structure and growth pattern may provide deeper insights into the origin(s) of this remarkable innovation.

\section{Biomed Central}


Angiosperm pollen tubes have a unique wall structure. Their thin growing tip is comprised almost entirely of pectins. Just behind the pectic tip, cellulose synthases operate to form a very thin, pecto-cellulosic primary wall. Then, still in the subapical region, $(1,3)-\beta$-glucan (callose) is synthesized beneath the thin primary wall to form a thick layer [8]. The mature pollen tube wall of most angiosperms is primarily made of callose ( $81 \%$ by weight in Nicotiana; ref. 9). As an amorphous polysaccharide, callose can be synthesized more rapidly than an equivalent weight of fibrous cellulosic cell wall [9] and it provides resistance to tensile and compression stress [10]. Callose also severely reduces wall permeability and since angiosperm pollen tube walls are also prone to forming septae ("callose plugs") [11], the plesiomorphic haustorial function of tubes is largely precluded. These patterns are general features of all angiosperms, from Amborella and water lilies to Arabidopsis and maize $[4,12]$. Yet, despite their ubiquity, the ancestral function of callose walls and plugs is not obvious. Tubes that lack callose in their walls retain their function in some derived eudicot lineages, such as Lamiales [13] and in an Arabidopsis mutant line [14,15], though they have reduced competitive ability in the latter [14].

Pollen tubes in ovules of gymnosperms rarely contain callose in lateral walls, and callose plugs have never been reported [16]. Callose is found in the tip wall of growing pollen tubes of some conifers [17], a pattern never seen in angiosperms. Studies of in vitro-grown gymnosperm pollen tubes do sometimes find callose (or mixed-glucans) in lateral tube walls $[17,18]$. Importantly, the deposition of callose is generally transient in gymnosperm male gametophytes and its extent and location varies even among closely related species [17-20]. Such transient and variable phenotypes contrast with the relatively invariant and persistent expression pattern seen in angiosperm pollen tubes.

Callose synthesis is mediated by the enzyme, callose synthase, encoded by the callose synthase gene, and originally described as a glucan synthase-like gene (GSL) in Nicotiana alata [21]. Hong et al. (2001) named the callose synthase gene family CalS after identifying 12 gene family members in Arabidopsis thaliana [22]. AtCalS5 and its characterized orthologues (NaGSL1 from $N$. alata) have been directly linked to pollen tube wall formation and callose plug deposition, as well as to pollen exine development $[14,15,23,24]$.

CalS5 appears to have an ancient origin by duplication. A comparative phylogenetic analysis of all CalS paralogs from the genomes of the moss, Physcomitrella patens [25] and Arabidopsis found that AtCalS5 was more closely related to a Physcomitrella CalS gene copy (PpCalS5) than to any other Arabidopsis paralog. Because callose was observed in the moss spore aperture region and
PpCalS5 was identified as a putative orthologue to AtCalS5, PpCalS5 was hypothesized to play a role in moss spore germination [25]. If so, then CalS5 involvement in pollen tube growth may ultimately derive from a more ancient function involving the germination process. As such, changes in gene regulation were likely prerequisites for the acquisition of novel callose deposition patterns in angiosperm pollen tube walls. Alternatively, the patterns arose via duplication and functional divergence of a CalS gene within seed plants, or perhaps within the stem lineage leading to angiosperms.

In this paper we present molecular evidence that CalS5 orthologues are expressed in mature pollen and pollen tubes of several extant early-diverging angiosperms in Nymphaeales and Austrobaileyales and likely also in Amborella trichopoda. CalS5 orthologues are also expressed in mature gymnosperm pollen, including one siphonagam (Gnetum) and one non-siphonogam (Ginkgo). In the siphonogamous conifer, Pinus, we report a potentially unique CalS gene expressed in germinated pollen. We discuss the implications of these findings for the evolution of the angiosperm pollen tube wall and suggest new avenues of research to clarify the functional roles of CalS in the seed plant male gametophyte.

\section{Results}

Putative orthologues of CalS5 are expressed in pollen and pollen tubes of early-diverging angiosperms

A nearly full length coding sequence was obtained from Cabomba caroliniana (Cabombaceae; Nymphaeales) (CcCalS5) comprising 5,562 bp which translated into a predicted 1854 amino acid polypeptide with $78 \%$ identity to Arabidopsis thaliana CalS5 (AtCalS5) and 66\% identity to the moss orthologue (PpCalS5) from the Physcomitrella patens genome [25]. The deduced polypeptide has a predicted topology containing between 13 and 17 transmembrane helices (as predicted by TMHMM v. 2.0; CBS, Lyngby, Denmark and SOSUI engine ver. 1.11; Nagoya University, Nagoya, Japan), a cytoplasmic N-terminal loop domain containing > 423 amino acids, and a large hydrophilic loop domain consisting of 758 amino acids, with loops between the helices ranging from 4 to 106 amino acids in length (Figure 1A).

RT-PCR was used to assess the presence of CcCalS5 transcript in various tissues of $C$. caroliniana, as well as in a pollen tube time-course experiment in Nymphaea odorata (Figure 1B) using intron-spanning primers. A 1,497 bp nucleotide sequence that shared $100 \%$ nucleotide identity with the CcCalS5 was amplified from $N$. odorata pollen and was used to designate the Nymphaea CalS5 orthologue (NoCalS5) in our RT-PCR experiments (Figure 1B). CcCalS5 expression was clearly observed in pre-dehiscent anthers and mature pollen of Cabomba (Figure 1B; left panel gel), but was also detected in low abundance in stem 


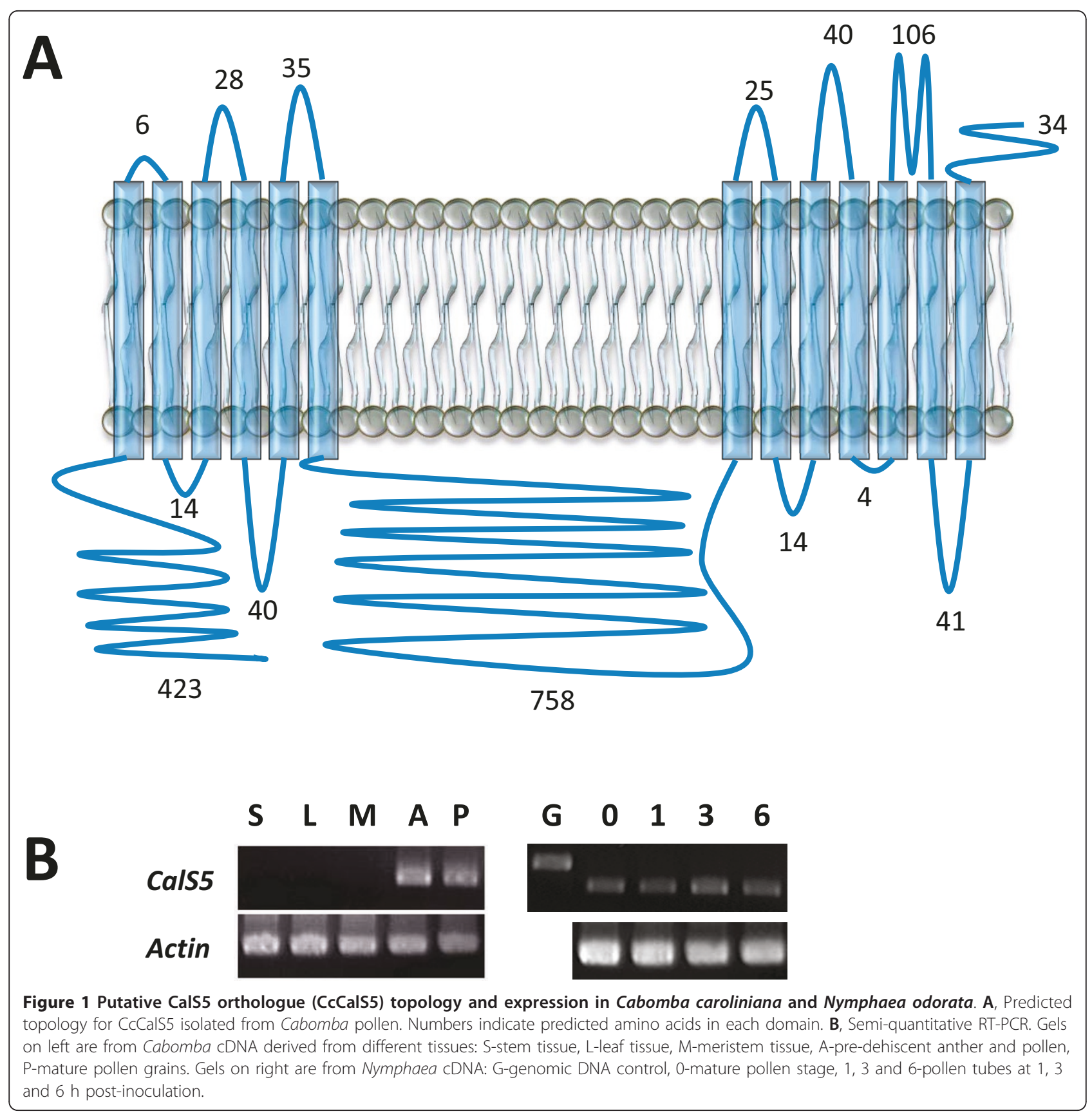

and leaf tissues during our RT-PCR optimization experiments (See Additional file 1). Expression of CcCalS5 in both Cabomba stem and leaf tissues was confirmed by sequencing clones of PCR products. NoCalS5 was consistently expressed during a time-course of in vitro-grown pollen tubes harvested at $0,1,3$ and 6 hrs after inoculation in liquid medium (Figure 1B; right panel gel).

In the 18-taxon phylogenetic tree constructed to infer orthologous relationships, all putative angiosperm CalS5 orthologues plus CcCalS5, formed a clade with $99 \%$ bootstrap support (Figure 2). A partial sequence from the putative CalS5 orthologue in Amborella trichopoda [26] also falls within the CalS5 clade (Figure 2). The putative CalS5 orthologues of Physcomitrella [25] and Selaginella (this study) are strongly supported as falling in an angiosperm clade of CalS paralogs, but not necessarily as sister to the CalS5 clade.

RT-PCR also recovered CalS transcripts from the mature pollen of the early-diverging angiosperms, Austrobaileya scandens (Austrobaileyaceae; Austrobaileyales), Nuphar advena (Nymphaeaceae; Nymphaeales), and Trithuria austinensis (Hydatellaceae; Nymphaeales). These 




Figure 2 Phylogenetic tree for full length CDSs of Arabidopsis, Physcomitrella CalS gene families and CalS5 orthologues. Phylogenetic tree based upon alignment of predicted polypeptides for full length CDSs of Arabidopsis, Physcomitrella Cals genes, and putative Cals orthologues identified in this study. Sequences for Amborella trichopoda CalS5 and Pinus taeda CalS13 are from partial CDNA fragments containing 196 and 471 amino acids, respectively. 
partial sequences from the hydrophilic loop domain align with the partial NoCalS5 sequence and are orthologous to CalS5, based on phylogenetic analysis (Additional files 2 and 3).

\section{Putative orthologues of CalS5 are expressed in pollen of Gnetum gnemon and Ginkgo biloba}

At $24 \mathrm{~h}$ after incubation, Ginkgo pollen stained for callose in the aperture area, intine, and also in the walls that separated prothallial, generative, and tube cells (Figure 3A). Gnetum pollen sheds its exine before tube growth, and prior to exine shedding, aniline blue staining was observed in the inner pollen wall (Figure 3B). After exine shedding, callose was not observed in the intine (Figure 3C, D).

Partial cDNA fragments from putative CalS5 orthologues were amplified from mature pollen of Gnetum gnemon (GgCalS5) and Ginkgo biloba (GbCalS5). Their predicted amino acid sequences aligned with the central loop domains of other CalS proteins, including those known to function during Arabidopsis pollen development (see Additional file 2). Phylogenetic analysis placed the Ginkgo and Gnetum sequences within a strongly supported clade of angiosperm CalS5 sequences (Additional file 3).

Interestingly, the aligned predicted protein sequences identified a short NASQ motif that Ginkgo shares with all members of Nymphaeales but that is absent from all other CalS5 sequences (see Additional file 2). Prosite scans of the aligned sequences identified the shared motif as a putative $\mathrm{N}$-glycosylation site (Prosite scan data not shown). Other putative functional motifs common within this alignment are CK2 and PKC phosphorylation sites that are highly conserved within all other Arabidopsis $\mathrm{CalS}$ sequences, however one predicted cAMP-dependent phosphorylation site, $\mathrm{K}(\mathrm{R} / \mathrm{K}) \mathrm{ES}$, was unique to most taxa within the CalS5 clade (see Additional file 2).

\section{A unique CalS orthologue is expressed in Pinus taeda pollen} A 1,413 bp CalS transcript was strongly expressed in mature and germinated Pinus taeda pollen (Figure 4A). Phylogenetic analysis of the 471 amino acid predicted polypeptide indicates $99 \%$ bootstrap support for its inclusion within a clade that does not include CalS5 (Figure 2). Because it is distantly related to any of the known Arabidopsis paralogs in that clade, we named the gene PtCalS13. PtCalS13 transcripts were abundant over a 72-hour time period of in vitro growth (Figure 4A), however repeated attempts to amplify CalS5-like transcripts over the same developmental stages failed. All combinations of primers that amplified CalS5-like fragments from Ginkgo, Gnetum, and early-divergent angiosperm pollen cDNA were attempted.
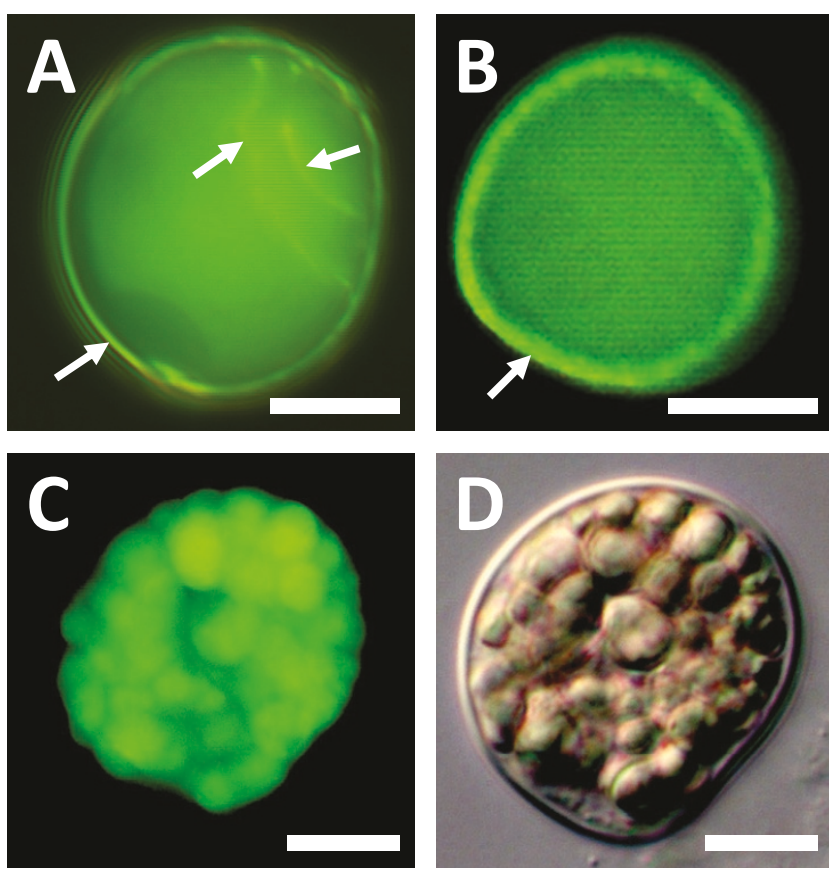

Figure 3 Cell wall staining in Ginkgo biloba and Gnetum gnemon. A, Ginkgo pollen grain showing aniline blue staining of internal male gametophyte walls and intine (arrows; one day of in vitro growth). B, Aniline blue stain localized to inner wall of Gnetum pollen (arrow) before exine shedding (one day of in vitro growth). C, D, Gnetum pollen grain after exine shedding (eight days of in vitro growth). C, Lack of aniline blue staining of intine (compare with DIC view in D). Note pollen is larger than first-day pollen in B and now contains abundant starch grains. Scale bars, $10 \mu \mathrm{m}$. 




Aniline blue staining was localized to the inner walls of the mature pollen grain, but not the tube wall (Figure 4BD). In pollen, strong staining was observed within the third intine layer at the proximal face of the microgametophyte, whereas weaker staining was apparent in the aperture region, the leptoma (Figure 4B). Upon germination, the aperture area became strongly stained, but the thick lateral walls of the pollen tube did not (Figure 4C, D). Calcofluor staining for cellulose was strong throughout all time periods observed during in vitro pollen tube growth (Figure 4E).

\section{Comparative analysis of predicted functional motifs among callose synthases}

Protein motif searches $[27,28]$ were performed on the sequences of CalS5 orthologues, as well as on all other callose synthases in Physcomitrella and Arabidopsis to evaluate conserved patterns of short linear motifs that may have functional significance. Comparison of the callose synthase protein sequences revealed conservation of seven different site patterns in all callose synthases: $\mathrm{N}$ glycosylation, cAMP- and cGMP-dependent protein kinase phosphorylation, protein kinase $\mathrm{C}$ phosphorylation (PKC), casein kinase II phosphorylation (CK2), tyrosine kinase phosphorylation, $\mathrm{N}$-myristoylation, and amidation (data not shown). The majority of putative functional motifs were found within the $\mathrm{N}$-terminal loop and the large central loop domain. The central loop domains of all callose synthases were consistently enriched with predicted PKC and CK2 phosphorylation sites. There were no clear distinguishing features of CalS5 orthologues with respect to selected patterns of 
predicted linear motifs when compared to the other callose synthases. Zea mays, Sorghum bicolor, and Selaginella moellendorfii all appear to have truncated CalS5 proteins, with the entire $\mathrm{N}$-terminal domains completely absent. This may reflect incorrect annotation, given the conserved nature of this large functional domain.

\section{In silico identification and phylogenetic analysis of putative CalS5 orthologues}

BLAST searches of NCBI [29] and Phytozome [30] databases revealed putative CalS5 orthologues from a phylogenetically diverse group of plant species which ranged from the unicellular green algae, Chlamydomonas reinhardtii to most completely sequenced angiosperms. Amino acid sequences from these putative orthologues were aligned with all known callose synthase gene family members from Physcomitrella, Arabidopsis, as well as other putative CalS5 orthologues for other angiosperms and the spikemoss, Selaginella moellendorfii. Also included in the alignment were two sequences of particular interest, the 471 amino acid PtCalS13 sequence obtained from Pinus taeda pollen cDNA, and a putative CalS5 orthologue identified in silico from the early-diverging angiosperm, Amborella trichopoda. The Amborella translated uniscript was obtained from the 454-EST build from the Ancestral Angiosperm Genome Project website [26] and comprised 196 amino acids. Several other translated uniscripts from Amborella displayed homology to CalS5, but because contig assembly was not feasible, they were not included in this analysis (data not shown).

\section{Discussion \\ CcCalS5 contains both general CalS functional motifs and CalS5-specific motifs}

All CalS proteins studied to date share a common topology with a large $\mathrm{N}$-terminal hydrophilic domain followed by two clusters of transmembrane (TM) domains that flank a large central hydrophilic domain [15,22,23,31,32]. The large hydrophilic loop is thought to accommodate interactions with other proteins, such as Rop1, UDP-glucose transferase (UGT1), sucrose synthase, and annexin, which enable the formation of a CalS enzyme complex [31]. Scans for functional motifs in CcCalS5 support the model of Verma and Hong [31], in which most known callose synthase proteins exhibit similarities in putative glycosylation and phosphorylation sites, particularly within the $\mathrm{N}$-terminal domain and the large central loop.

With respect to pollen tube growth, cAMP is a known signalling molecule for pollen tube guidance and growth [33]. In our alignment of the loop domains, we identified one putative cAMP and cGMP-dependent phosphorylation site common to all seed plant members of the CalS5 clade, but absent from PpCalS5 of Physcomitrella and from all other CalS paralogs (see Additional file 2). We also identified a putative CalS5 N-glycosylation site unique to seed plants - present in Ginkgo and all members of the Nymphaeales clade (see Additional file 2) but absent from Austrobaileya and all monocots and eudicots. It is not known whether these variants cause functional differences in CalS5 expression.

\section{CalS genes involved in male gametophyte development}

Five of 12 callose synthase genes (CalS5, CalS9, CalS10, CalS11, and CalS12) have been shown to function during microsporogenesis and microgametogenesis in Arabidopsis [15,34-37]. In contrast to other CalS genes, CalS11 and CalS12 contain only two or three exons [22] and are $\mathrm{Ca}^{2}$ ${ }^{+}$-dependent [38]. CalS11 and CalS12 are genetically linked and perform partially redundant roles in the formation of walls separating microspores of the tetrad and in late maturation of the male gametophyte [34]. CalS12 is also activated during wound response and on stigmatic papillae [39].

The other $10 \mathrm{CalS}$ genes contain between 39 and 50 exons and are $\mathrm{Ca}^{2+}$-independent $[40,41]$. CalS5 is sporophytically expressed to form the callose wall of pollen mother cells and microspore tetrads and is also the predominant gametophytically-expressed transcript in germinating pollen and growing pollen tubes [14, 15, this study]. CalS9 and CalS10 function early in microgametogenesis $[35,36]$ since mutant lines independently exhibited functional aberrations during the entry of microspores into mitosis. Mutants of CalS9 caused failure of mitosis II as well as abnormal positioning of nuclei in mature pollen and precocious germination, inside the anther [37]. CalS10 is known to be involved in cell plate formation [22]. Silencing of CalS9 and CalS10 using gene-specific dsRNAi constructs also resulted in a dwarfed growth habit, suggesting that both also function in the sporophytic phase [35]. These studies show that male gametophyte development in Arabidopsis is mediated by transient expression of a number of CalS genes, whereas in all angiosperms studied to date CalS5 is abundant and predominant during pollen germination and in all stages of pollen tube growth.

\section{The Pinus taeda male gametophyte expresses a unique Cals gene in pollen}

We found strong expression of a novel CalS gene in mature and in germinated pollen of P. taeda. PtCalS13 was strongly supported as falling within a clade of AtCalS paralogs that does not include any of the known gametophytically-expressed paralogs (AtCalS5 and AtCalS9-12). PtCalS13 cannot be a deeply divergent copy of CalS5 or CalS9-12, but is most likely a novel copy of CalS (alternatively, it may be a deeply divergent orthologue of CalS1-4 or CalS6-8; Figure 2). CalS5 transcripts were not found at the same stages of development, suggesting 
PtCalS13 functions in place of CalS5. However, more work is needed to test this hypothesis.

Pinus species are quite variable in the location and extent of pollen callose deposition [42,43]. In P. taeda, callose was present in the intine and male gametophyte walls before pollen germination. Upon germination, it became strongly expressed in the aperture region surrounding the exiting pollen tube, but did not extend into the tube wall, as it does in angiosperms $[41,44,45]$. Thus, the callose distribution pattern associated with PtCalS13 expression is quite different from the CalS5 pattern in germinating angiosperm pollen.

\section{Ginkgo and Gnetum express an orthologue of CalS5 in pollen}

There is strong evidence that the CalS genes expressed in mature pollen of Gnetum and Ginkgo are orthologous to angiosperm CalS5 since their protein sequences were nested within the angiosperm CalS5 clade. Callose deposition is apparently restricted to pollen in these species. In Gnetum it was present in the inner wall of pollen before exine shedding, but after shedding it was absent from the intine which is continuous with the emerging pollen tube wall. In Ginkgo, callose was abundant in the intine and in the walls of the male gametophyte. A study of in vitro pollen tube growth in Ginkgo found that their tube walls stained weakly for aniline blue but reacted strongly to calcofluor white and $\beta-(1,3)(1,4)$ antibodies, suggesting the presence of $\beta$ - $(1,3)(1,4)$ mixed glucans [18].

\section{The evolutionary developmental origins of callose synthase expression in angiosperm pollen tubes}

Fast growing pollen tubes are arbiters of the rapid reproductive cycles of angiosperms and their unique wall structure may have been a trigger for extensive pollen tube growth rate evolution in the group $[1,4,46]$. Gene family expansions are thought to have been important for trait diversification early in angiosperm history or pre-history $[47,48]$. Six CalS paralogs from three ancient land plant lineages - five from Arabidopsis [22] and one from Pinus (this study) - are now known to be expressed in male gametophytes of seed plants (Figure 5). To date, only CalS5 has been shown to be expressed in pollen tubes, and only in a few model system eudicots $[15,23,24]$. The finding that pollen tubes of a broad set of extant earlydiverging angiosperms also utilize CalS5 in their pollen tubes supports the homology of callose walls and plugs in flowering plants $[4,5]$.

CalS5 transcripts were also found in mature and/or germinating pollen, of two distantly-related gymnosperms, Ginkgo and Gnetum. The predominant anatomical location of callose in Ginkgo was in the intine and internal gametophytic cell walls, whereas the pollen tube-forming intine of germinated Gnetum pollen did not contain callose. In all early-divergent angiosperms used in this study, callose was present within the intine of germinating pollen and continuous with the callose inner wall of the pollen tube $[4,43,49]$. Gametophytic expression of CalS5 has a similar pattern in Arabidopsis and tobacco $[14,15,23,24,32,37,50]$. Thus, it seems likely that CalS5 had an ancestral expression pattern within the inner pollen wall that later became modified via the evolution of gene regulation to function in growing pollen tubes of an ancestor of extant angiosperms (Figure 5).

A number of hypotheses have been proposed as to what the ancestral function of callose in germinating pollen or spores might have been. In the moss, Physcomitrella, callose was deposited in the inner exine layer (not the intine) near the aperture at the proximal pole of the spore just before germination [25]. Callose was inferred to function in spore germination and it was suggested that a CalS5 orthologue was involved [25]. Expression data are needed to determine if these results reflect an ancient aspect of land plant spore germination or an apomorphic feature of moss spores (Figure 5). We also found callose thickenings in the aperture area of germinating Pinus pollen, but these were associated with PtCalS13, not a CalS5 orthologue (Figure 5). Callose is localized to the inner intine of mature pollen of some Pinus species [51-53], the outer layer in P. sylvestris $[17,54]$, and is absent from the intine of P. wilsonii [55]. Pacini et al. (1999) concluded that such variable and transient expression of callose in Pinus pollen indicates that it functions as a reserve polysaccharide, rather than serving a structural or prophylactic function [45]. Górska-Brylass (1970) argued its presence in the proximal outer intine was due to non-retrieval of callose plates from the prior divisions of prothallial cells [56]. Alternatively, its co-localization with degenerate prothallial cells may indicate the prior involvement of callose as a wall sealant that initiates cell death, a pattern also seen during megasporogenesis in seed plants [57].

To date no study has convincingly shown callose to be the predominant and permanent constituent of any gymnosperm pollen tube wall, nor is there any finding of a callose plug in a gymnosperm pollen tube. For example, callose is reported from young but not old tubes in Pinus and Cycas $[17,20]$; it is a transient feature of long-lived Pinus tubes during winter dormancy [17]; and it can appear at the growing tube tip but does not persist in the lateral walls as the tip continues its growth $[16,17]$. From a functional standpoint, one should note that the origin of persistent callose walls and plugs in angiosperm pollen tubes must have been contingent on a shift from a primarily haustorial function, such as occurs in root hairs, rhizoids and pollen tubes of Cycads and Ginkgo, to a new one in which a short-lived and fast-growing tip functions primarily to carry sperm to egg. Pollen tubes of gymnosperms are long-lived and extend from a multicellular 


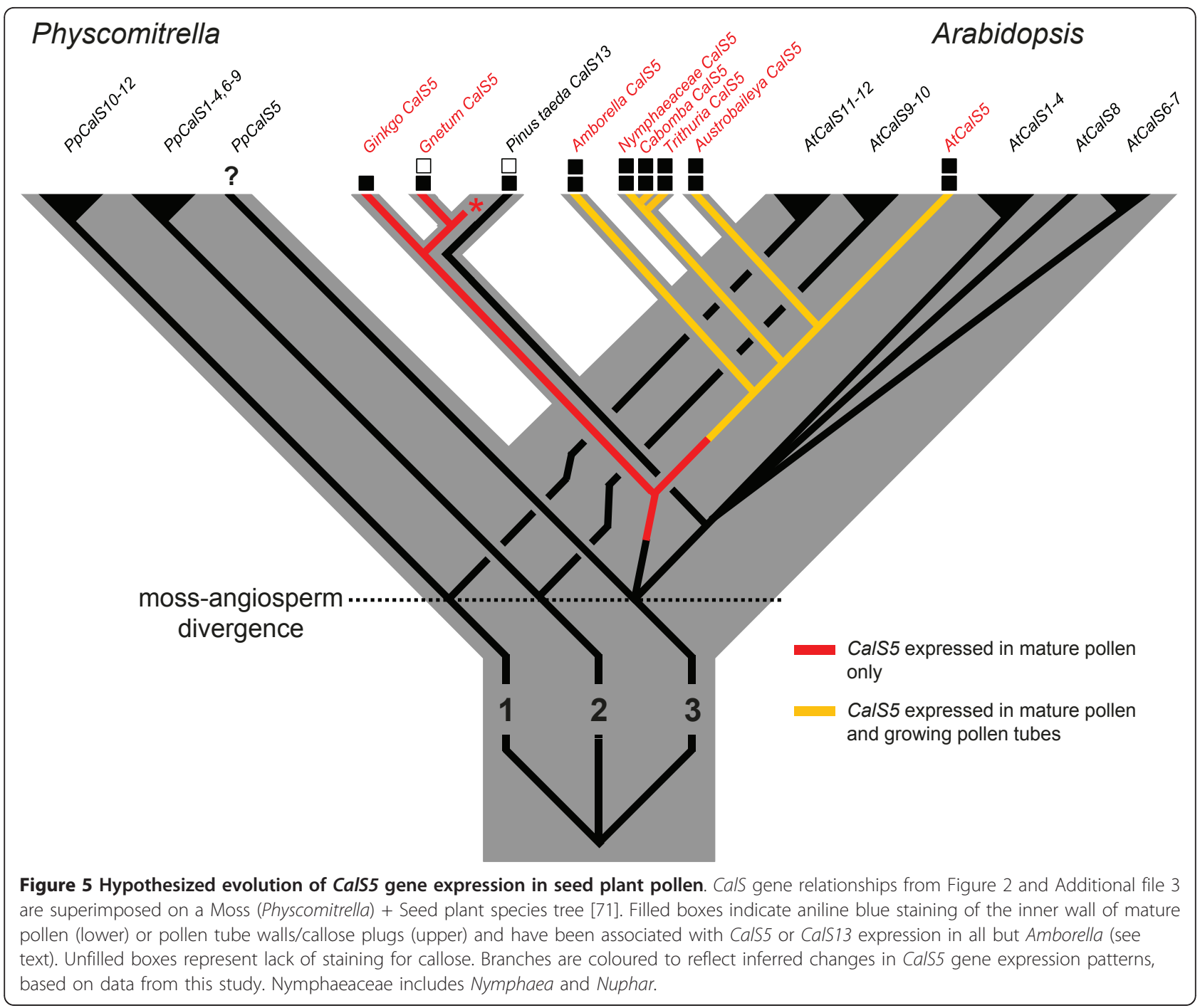

male gametophyte, which typically lives within a pollen grain attached to female tissues at the pollination site [3]. In most conifers, sperm are formed late in male gametophyte ontogeny and must travel from the pollen grain to the tip late in life. Thus, the biology of the fertilization process in most gymnosperms prevents their pollen tubes from utilizing callose as a semi-permanent structural feature of their walls.

At some point(s) along the lineage leading to angiosperms, two shifts in CalS5 localization occurred - callose deposition became restricted to a short subapical region of the growing pollen tube tip and to a small distal region of the tube where a callose plug forms. Importantly, both of these changes in localization must also have involved changes in callose retrieval, giving rise to persistent callose walls and plugs. One model for the origin of angiosperm pollen tube morphology is that an ancestral conifer-like pollen tube became transformed by the evolution of faster growth rates [46], causing the callose synthesis and retrieval machinery to be displaced from the newly-forming tube tip to a subapical position $[1,17]$. Comparative analyses support the notion that early angiosperm pollen tubes grew faster than those of their gymnosperm-like ancestor (extant gymnosperm pollen tubes are characterized by exceptionally slow pollen tube growth rates) [5]. What is not clear is whether nonretrieval of callose is a cause or a consequence of the origin of faster growth rates.

Given the increased tendency of molecular phylogenetic analyses to place conifers/Gnetales in an isolated position relative to angiosperms [58], it is worth considering that angiosperm pollen tube morphology may have evolved independently from an ancestral Ginkgo/Cycadlike haustorial tube [20], rather than from a transitional siphonogamous, conifer-like predecessor. Resolving the question of pollen tube origins will require careful 
developmental analyses of pollen tube growth and the many genes that mediate differences among extant seed plant groups. It would be especially interesting to look at the evolution of the callase ( $\beta$-1,3-glucanase) gene family, which catalyzes the retrieval of callose [59].

\section{Conclusion}

This study supports the homology of callose pollen tube walls and plugs across flowering plants at the level of CalS5 gene expression. Since CalS5 was also found to be actively transcribed in mature pollen of Ginkgo and Gnetum, we suggest that CalS5 localization to the inner intine of mature germinating pollen was present in a distant angiosperm ancestor, perhaps extending into the walls of young pollen tubes, and was later co-opted as a non-transient feature of angiosperm pollen tubes (Figure 5). CalS5 is a structural gene that originated by duplication long before the origin of extant angiosperms. Thus, the novel callose deposition patterns of angiosperm pollen tubes must be a consequence of the evolution of novel regulation of an ancient gene. It remains to be seen to what extent this involved duplication and divergence of other genes involved in the callose synthesis or retrieval pathways, and to what degree it was or was not a developmental outcome of the evolution of faster growth rates.

\section{Methods}

\section{Plant material}

Whole flowers from Austrobaileya scandens White were collected near Millaa Millaa, Queensland, Australia (17 $\left.31^{\prime} 15^{\prime \prime} \mathrm{S}, 145^{\circ} 33^{\prime} 53^{\prime \prime} \mathrm{E}\right)$. A scandens pollen tubes were grown in hanging drops of BK media [60] containing 2.5\% sucrose inside closed petri plates for 5 to 12 hours. Pollen from Trithuria austinensis Sokoloff was collected in Branchinella Lake, shire of Manjimup, Western Australia (34 $\left.16^{\prime} \mathrm{S}, 116^{\circ} 42^{\prime} \mathrm{E}\right)$. Pollen or pollen tubes of these two species were centrifuged briefly and resuspended in RN Later (Ambion, Austin, TX, USA) and RNA was isolated within several weeks. RNA was isolated from fresh pollen or pollen tubes for the remaining species below. Pollen from Ginkgo biloba L. was collected from trees growing on the University of Tennessee campus in Knoxville, TN, USA. Nuphar advena Aiton. flowers were collected near Sparta, TN, USA ( $\left.35^{\circ} 55^{\prime} 11^{\prime \prime} \mathrm{N}, 85^{\circ} 20^{\prime} 41^{\prime \prime} \mathrm{W}\right)$ and pollen tubes were grown in BK media with $5 \%$ sucrose for two hours before RNA isolation. Nymphaea odorata Aiton. flowers were collected from a pond in Knoxville, TN, USA ( $35^{\circ} 53^{\prime}$ $51^{\prime \prime} \mathrm{N}, 84^{\circ} 10^{\prime} 23^{\prime \prime}$ W). Cabomba caroliniana A. Gray plants were grown in greenhouse water tanks and were originally collected from Racoon Creek, Jackson County, AL, USA ( $\left.34^{\circ} 46^{\prime} \mathrm{N}, 85^{\circ} 50^{\prime} \mathrm{W}\right)$ or purchased from Carolina Biological Supply (Burlington, NC, USA). Gnetum gnemon L. flowers with dehiscent anthers were collected from greenhouse-grown plants in DEPC-treated water, vortexed to separate pollen from all other flower parts, and briefly centrifuged prior to RNA isolation. Pollen from Pinus taeda L. and P. strobus L. was collected from trees on the University of Tennessee campus. Pinus pollen was grown in a liquid medium containing $10 \%$ sucrose, $15 \mathrm{mM}$ MES, $1 \mathrm{mM} \mathrm{H}_{3} \mathrm{~B}, 1 \mathrm{mM} \mathrm{CaCl}_{2}$, $\mathrm{pH} 4.0$ in petri plates sealed with parafilm.

\section{In vitro pollen tube experiments with Nymphaea and Cabomba}

Due to the poor germination observed for both Nymphaea and Cabomba pollen in standard BK media, stigmatic fluid from first day Nymphaea flowers was collected on site with a disposable pipette and used as the pollen tube growth medium for these species. Both species exhibited 70 to $90 \%$ germination success when grown in the fresh stigmatic fluid, and thus fresh stigmatic fluid was used for all pollen tube experiments described here. Stigmatic fluid was collected shortly after flower opening (9 to $10 \mathrm{am}$ ), and centrifuged for three minutes at 13,000 rpm to remove any contaminating debris and/or pollen grains. Anthers from Cabomba and Nymphaea were removed with forceps and placed into $1.7 \mathrm{ml}$ tubes that contained $1 \mathrm{ml}$ of stigmatic fluid. Anther number was used to standardize samples for pollen density during tube growth. Tubes were vortexed briefly to separate pollen grains from anthers, anthers were then removed, and contents were transferred to a small petri plate for pollen tube growth at room temperature. Nymphaea pollen tubes were grown for various lengths of time (one, three, and six hours postinoculation) for gene expression experiments. For RNA extractions, pooled pollen tube samples were harvested at each time point, centrifuged at a 2,000 rpm for 30 seconds to maintain pollen tube integrity, and immediately frozen in liquid nitrogen after growth medium was removed.

\section{Bioinformatics and primer design}

In order to search for orthologous CalS5 gene sequence in the early-diverging angiosperms, the local BLAST tool on the ancestral angiosperm genome project website [26] was used to blast the Arabidopsis CalS5 protein sequence (tBlastn) against all available 454-Sanger hybrid databases. After performing non-redundant nucleotide NCBI BLAST searches of individual uniscript hits, primers were designed to amplify a Nuphar advena uniscript sequence (c78546) with the lowest E-value corresponding to AtCalS5. The primers designed towards Nuphar advena sequence (Sec16F; Sec17R) amplified a $600 \mathrm{bp}$ highly conserved region of sequence within the predicted hydrophilic domain of the putative CalS5 orthologue. These primers were also used for amplifying putative CalS5 orthologous pollen-derived cDNA from Ginkgo biloba, Austrobaileya scandens, and the more closely related water lily species, Cabomba caroliniana, 
Nymphaea odorata, and Trithuria austinensis. Another forward primer (Sec17F) nested within this sequence and the reverse primer (Sec17R) enabled PCR amplification of the $250 \mathrm{bp}$ Gnetum sequence. Various multiple sequence alignments were carried out on all callose synthase DNA and protein sequences in Physcomitrella patens and Arabidopsis to aid in primer selection. To amplify the Pinus taeda CalS cDNA, two EST sequences showing the highest homology to AtCalS5 and PpCalS5 sequence (AI812992 and FJ114840) obtained from nonredundant NCBI BLAST of Pinus were aligned with PpCalS5 and AtCalS5. Forward FJ114840-F and reverse primer AI812992-R amplified a product that reflected the alignment. To prevent amplification of other pollenexpressed CalS genes (CalS9, CalS10, CalS11, CalS12), particular attention was given to these sequences during primer design for PCR applications. All primer design and sequence alignments were performed using Vector NTI software (Invitrogen, Carlsbad, CA, USA). A complete set of primers used in this study is listed in Additional file 4.

\section{Molecular analyses}

Total RNA from pollen and pollen tubes was isolated using Tri Reagent ${ }^{\top}$ (Ambion, Austin, TX, USA) and a modified CTAB protocol was required for Cabomba vegetative tissues [61]. For cDNA synthesis, $1.5 \mu \mathrm{g}$ of total RNA was used, according to the manufacturer's protocol (ArrayScript; Ambion, Austin, TX, USA). Prior to cDNA synthesis, all samples were subjected to DNase I (TurboFree DNase kit; Ambion). RNA was assessed for quality with agarose gel electrophoresis and quantified with a NanoDrop (Thermo Scientific, Waltham, MA, USA) spectrophotometer. A 3' RACE procedure was used according to the First Choice RLM RACE kit (Ambion, Austin, TX, USA) to acquire the 3' end of the Cabomba caroliniana CalS5 gene. However, due to difficulties with the 5 ' RACE protocol 5' products were amplified using a forward primer designed towards highly conserved sequence within a multiple sequence alignment of CalS5 orthologues (CalS515F). Inverse PCR was performed to obtain the 5' end of the full length cDNA using a digest/re-ligation/ digest strategy with HindIII/PstI, respectively [62]. Although attempts to obtain the 5 ' end of the cDNA were incomplete, the inverse PCR procedure did enable the sequencing of introns that flanked the 5'-most exon. Identification of these intron-exon boundaries enabled the design of intron-spanning primers for semi-quantitative RT-PCR. All PCR reactions that required cloning were performed with Herculase II DNA fusion polymerase (Agilent, Santa Clara, CA, USA). Products were gel purified using a Qiaex II gel purification kit (Qiagen, Valencia, CA), cloned into pcr8-GW-TOPO (Invitrogen, Carlsbad, CA, USA), and sequenced on an ABI 3100 capillary sequencer at the University of Tennessee Molecular Biology Resource Facility. cDNA tissue sources are listed in Additional file 5. All sequences were deposited in Genbank [http://www.ncbi.nlm.nih.gov/genbank/index.html].

\section{Semi-quantitative RT-PCR}

To prevent amplification of possible orthologous pollenexpressed CalS genes (CalS9, CalS10, CalS11, CalS12), both protein and nucleotide alignments were used to design primers to amplify a $631 \mathrm{bp}$ intron-flanking sequence that shared a predicted low homology to nontarget CalS cDNA. Primers were designed to amplify an Actin gene isolated from Nuphar advena pollen. Actin was also used as a reference gene to confirm equal template loading in Pinus taeda RT-PCR. A master mix of PCR reagents (described above) was used to amplify the CalS5 fragment and Actin control gene in separate reactions at equal template concentrations and cycling parameters. Threshold cycle optimization was determined by performing PCR amplifications over a range of cycles. The number of PCR cycles selected corresponded to where the trend line exhibited the highest correlation to exponential amplification. Bands representing each tissue type were purified, cloned, and the sequence was verified to confirm single product amplification.

\section{Phylogenetic analysis}

Alignments of predicted amino acid sequences were performed using MAFFT (Cambridge, Engand) [63]. Mesquite (Vancouver, BC, Canada) [64] was used to truncate taxon names. Gblocks software (Barcelona, Spain) [65] with gap mode ALL was used to exclude poorly aligned regions. Model testing was performed using ProtTest 2.4 (Vigo, Spain) [66] using only those substitution models present in RAxML (San Diego, CA, USA) $[67,68]$. RAxML was used on the CIPRES web server $[68,69]$ to infer phylogenetic trees and do fast bootstrapping (maximum likelihood method). Trees were visualized using FigTree (Edinburgh, Scotland, UK) [70].

\section{Additional material}

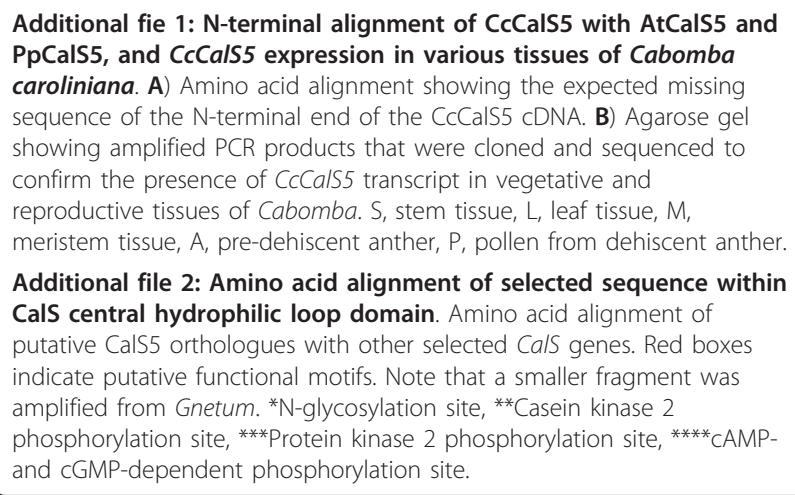


Additional file 3: Phylogenetic tree from central loop domains of Arabidopsis and Physcomitrella CalS genes and putative CalS5 orthologues. Phylogenetic tree based on alignment of predicted polypeptides for central loop domains of known Arabidopsis, Physcomitrella Cals genes and putative Cals orthologues identified in this study.

Additional file 4: Primers used in this study. Primers used to amplify Cals orthologues and conduct semi-quantitative RT-PCR.

Additional file 5: Sources for putative CalS orthologues amplified in this study. Tissue sources and CDNA fragment sizes for putative CalS orthologues amplified from taxa in this study.

\section{Abbreviations}

AsCalS5: Austrobaileya scandens CalS5; CalS: callose synthase; CK2: casein kinase II; PKC: protein kinase C; CcCalS5: Cabomba caroliniana CalS5; GbCalS5: Ginkgo biloba CalS5; GgCalS5 Gnetum gnemon CalS5; GSL: glucan synthase-like; NoCalS5: Nymphaea odorata CalS5; PtCalS13: Pinus taeda CalS13; TaCalS5: Trithuria austinensis CalS5; UGT1: UDP-glucose transferase I.

\section{Acknowledgements}

The authors wish to thank Richard Moore, Barry Bruce and three anonymous reviewers for their insightful comments. For technical assistance we thank Mark Lazzaro (gymnosperm pollen tube growth), Ken McFarland (greenhouse), Joe May (sequencing of clones), Nick Buckley (lab assistance) and Karen Hughes (use of lab equipment). We also thank Mackenzie Taylor for Trithuria austinensis samples and David McFarland for access to the Nymphaea odorata population. The work of all authors was supported by US National Science Foundation awards DEB 0640792 and IOS 1052291 to J. H. W.

\section{Authors' contributions}

JA conceived of the study, carried out the experiments, and drafted the manuscript. AM performed troubleshooting of RNA isolation protocols and assisted in experiments. BO performed phylogenetic analyses and tree construction. JW also conceived of the study, provided experimental guidance, and shared in writing the manuscript. All authors read and approved the final manuscript.

\section{Competing interests}

The authors declare that they have no competing interests.

Received: 1 February 2011 Accepted: 1 July 2011 Published: 1 July 2011

\section{References}

1. Knox RB: Pollen-pistil interactions. In Cellular Interactions. Edited by: Linskens HF, Heslop-Harrison J. Berlin, Germany: Springer-Verlag; 1984:77-92.

2. Pettitt JM: Detection in primitive gymnosperms of proteins and glycoproteins of possible significance in reproduction. Nature 1977, 266:530.

3. Friedman WE: The evolutionary history of the seed plant male gametophyte. Trends Ecol Evol 1993, 8:15-21.

4. Williams JH: Novelties of the flowering plant pollen tube underlie diversification of a key life history stage. Proc Natl Acad Sci USA 2008, 105:11259-11263.

5. Williams JH: Amborella trichopoda (Amborellaceae) and the evolutionary developmental origins of the angiosperm progamic phase. Am J Bot 2009, 96:144-165.

6. Gould SJ, Vrba ES: Exaptation: a missing term in the science of form. Paleobiology 1982, 8:4-15.

7. Friedman WE, Floyd SK: Perspective: the origin of flowering plants and their reproductive biology - a tale of two phylogenies. Evolution 2001, 55:217-231.

8. Meikle PJ, Bonig I, Hoogenraad NJ, Clarke AE, Stone BA: The location of $1,3-\beta$-glucans in the walls of pollen tubes of Nicotiana alata using a 1,3$\beta$-glucan-specific monoclonal antibody. Planta 1991, 185:1-8.

9. Schlupmann H, Bacic A, Read SM: Uridine diphosphate glucose metabolism and callose synthesis in cultured pollen tubes of Nicotiana alata Link et Otto. Plant Phys 1994, 105:659-670.
10. Parre E, Geitmann A: More than a leak sealant. The mechanical properties of callose in pollen tubes. Plant Physiol 2005, 137:274-286.

11. Mogami N, Miyamoto M, Onozuka M, Nakamura N: Comparison of callose plug structure between dicotyledon and monocotyledon pollen germinated in vitro. Grana 2006, 45:249-256.

12. Mascarenhas JP: Molecular mechanisms of pollen tube growth and differentiation. Plant Cell 1993, 5:1303-1314.

13. Prósperi $\mathrm{CH}$, Coccuci AE: Importancia taxonomica de la calosa de los tubos polinicos en Tubiflorae. Kurtziana 1979, 12-13:75-81.

14. Nishikawa S, Zinkl GM, Swanson RJ, Maruyama D, Preuss D: Callose (beta1,3 glucan) is essential for Arabidopsis pollen wall patterning, but not tube growth. BMC Plant Biol 2005, 5:22.

15. Dong X, Hong Z, Sivaramakrishnan M, Mahfouz M, Verma DP: Callose synthase (CaIS5) is required for exine formation during microgametogenesis and for pollen viability in Arabidopsis. Plant J 2005 42:315-328.

16. Fernando DD, Quinn CR, Brenner ED, Owens JN: Male gametophyte development and evolution in extant gymnosperms. Int/ I Plant Dev Biol 2010, 4:47-63.

17. Derksen J, Li YQ, Knuiman B, Guerts H: The wall of Pinus sylvestris pollen tubes. Protoplasma 1999, 208:26-36.

18. Yatomi R, Nakamura S, Nakamura N: Immunochemical and cytochemical detection of wall components of germinated pollen of gymnosperms. Grana 2002, 41:21-28.

19. Martens $P$, Waterkeyn L: Structure du pollen "ailé" chez les Coniféres. Cellule 1962, 62:173-222.

20. Pettitt JM: Ultrastructural and immunocytochemical demonstration of gametophytic proteins in the pollen tube wall of the primitive gymnosperm Cycas. J Cell Sci 1982, 57:189-213.

21. Saxena IM, Brown RMJ: Cellulose synthases and related enzymes. Curr Opin Plant Biol 3:523-531.

22. Hong Z, Delauney AJ, Verma DP: A cell plate-specific callose synthase and its interaction with phragmoplastin. Plant Cell 2001, 13:755-768.

23. Brownfield L, Ford K, Doblin MS, Newbigin E, Read S, Bacic A: Proteomic and biochemical evidence links the callose synthase in Nicotiana alata pollen tubes to the product of the NaGSL1 gene. Plant J 2007, 52:147-156.

24. Brownfield L, Wilson S, Newbigin E, Bacic A, Read S: Molecular control of the glucan synthase-like protein NaGSL1 and callose synthesis during growth of Nicotiana alata pollen tubes. Biochem J 2008, 414:43-52.

25. Schuette S, Wood AJ, Geisler M, Geisler-Lee J, Ligrone R, Renzaglia KS: Novel localization of callose in the spores of Physcomitrella patens and phylogenomics of the callose synthase gene family. Ann Bot 2009, 103:749-756.

26. Ancestral angiosperm genome project. [http://ancangio.uga.edu/]

27. PBIL Network protein sequence analysis. [http://expasy.org/tools/ scanprosite/].

28. Falquet L, Pagni M, Bucher P, Hulo N, Sigrist CJ, Hofmann K, Bairoch A: The PROSITE database, its status in 2002. Nucleic Acids Res 2002, 30:235-238.

29. National Center for Biotechnological Information. [http://www.ncbi.nlm. nih.gov/].

30. Phytozome: a tool for green plant comparative genomics. [http://www. phytozome.org].

31. Verma DP, Hong Z: Plant callose synthase complexes. Plant Mol Biol 2001, 47:693-701.

32. Doblin MS, De Melis L, Newbigin E, Bacic A, Read SM: Pollen tubes of Nicotiana alata express two genes from different $\beta$-glucan synthase families. Plant Phys 125:2040-2052.

33. Mountinho A, Hussey PJ, Trewavas AJ, Malho R: cAMP acts as a second messenger in pollen tube growth and reorientation. Proc Natl Acad Sci USA 2008, 98:10481-10486.

34. Enns LC, Kanaoka MM, Torii KU, Comai L, Okada K, Cleland RE: Two callose synthases, GSL1 and GSL5, play an essential and redundant role in plant and pollen development and in fertility. Plant Mol Biol 2005, 58:333-349.

35. Toller A, Brownfield L, Neu C, Twell D, Schulze-Lefert P: Dual function of Arabidopsis glucan synthase-like genes GSL8 and GSL10 in male gametophyte development and plant growth. Plant J 2008, 54:911-923.

36. Huang L, Chen XY, Rim Y, Han X, Cho WK, Kim SW, Kim JY: Arabidopsis glucan synthase-like 10 functions in male gametogenesis. $J$ Plant Physiol 2009, 166:344-352. 
37. Xie B, Wang $X$, Hong Z: Precocious pollen germination in Arabidopsis plants with altered callose deposition during microsporogenesis. Planta 2010, 231:809-823.

38. Delmer DP: Cellulose biosynthesis. Annu Rev Plant Physiol 1987, 38:259-290

39. Jacobs AK, Lipka V, Burton RA, Panstruga R, Strizhov N, Schulze-Lefert P, Fincher GB: An Arabidopsis Callose Synthase, GSL5, Is Required for Wound and Papillary Callose Formation. Plant Cell 2003, 15:2503-2513.

40. Li YQ, Moscatelli A, Cai G, Cresti M: Functional interactions among cytoskeleton, membranes, and cell wall in the pollen tube of flowering plants. Int Rev Cytol 1997, 176:133-199.

41. Schlupmann H, Bacic A, Read SM: A novel callose synthase from pollen tubes of Nicotiana. Planta 1993, 191:470-481.

42. Ferguson C, Teeri TT, Siika-aho M, Read SM, Bacic A: Location of cellulose and callose in pollen tubes and grains of Nicotiana tabacum. Planta 1998, 206:452-460

43. Williams JH, McNeilage RT, Lettre MT, Taylor ML: Pollen tube growth and the pollen-tube pathway of Nymphaea odorata (Nymphaeaceae). Bot J Lin Soc 2010, 162:581-593.

44. Heslop-Harrison J: Aspects of the structure, cytochemistry and germination of the pollen of rye (Secale cereale L.). Ann Bot 1979, 44:1-47.

45. Pacini E, Franchi GG, Ripaccioli M: Ripe pollen structure and histochemistry of some gymnosperms. Plant Syst Evol 1999, 217:81-99.

46. Mulcahy DL: The rise of angiosperms: a genecological factor. Science 1979, 206:20-23.

47. Soltis DE, Albert VA, Leebens-Mack J, Bell CD, Paterson AH, Zheng CF, Sankoff D, dePamphilis CW, Wall PK, Soltis PS: Polyploidy and angiosperm diversification. Am J Bot 2009, 96:336-348.

48. Van de Peer $Y$, Fawcett JA, Proost $S$, Sterck $L$, Vandepoele $K$ : The flowering world: a tale of duplications. Trends Plant Sci 2009, 14:680-688.

49. Taylor ML, Williams $\mathrm{JH}$ : Consequences of pollination syndrome evolution for post-pollination biology in an ancient angiosperm family. Int J Plant Sci 2009, 170:584-598.

50. Cai G, Faleri C, Del Casino C, Emons AM, Cresti M: Distribution of callose synthase, cellulose synthase and sucrose synthase in tobacco pollen tube is controlled in dissimilar ways by actin filaments and microtubules. Plant Physiol 2010, 155:1169-1190.

51. Pettitt JM: Pollen tube development and characteristics of protein emission in conifers. Ann Bot 1985, 56:379-397.

52. Waterkeyn L: Callose microsporocyteaire et callose pollinique. In Pollen Physiology and Fertilization. Edited by: Linskens HF. London, Amsterdam: N Holland Publishing; 1964:52-58.

53. Martens P, Waterkeyn L, Huyskens M: Organization and symmetry of microspores and origin of intine in Pinus sylvestris. Phytomorph 1967, 17:114-118.

54. Rowley JR, Skvarla JJ, Walles B: Microsporogenesis in Pinus sylvestris L. VIII. Tapetal and late pollen grain development. Plant Syst Evol 2000, 225:201-224.

55. Fang KF, Wang YN, Yu TQ, Zhang LY, Baluska F, Samaj J, Lin JX: Isolation of de-exined pollen and cytotogical studies of the pollen intines of Pinus bungeana Zucc. Ex Endl. and Picea wilsonii Mast. Flora 2008, 203:332-340.

56. Górska-Brylass A: The "callose stage" of the generative cells in pollen grains. Grana 1970, 10:21-30.

57. Wu H, Cheung AY: Programmed cell death in plant reproduction. Plant Mol Bio 2000, 44:267-281.

58. Mathews S, Clements MD, Beilstein MA: A duplicate gene rooting of seed plants and the phylogenetic position of flowering plants. Phil Trans Roy Soc B 2010, 365:383-395

59. Albersheim P, Darvill A, Roberts K, Sederoff R, Staehelin A: Plant Cell Walls: from Chemistry to Biology New York, NY: Garland Science; 2011.

60. Brewbaker JL, Kwak BH: The essential role of calcium ion in pollen germination and pollen tube growth. Am J Bot 1963, 50:859-865.

61. Gasic K, Hernandez A, Korban S: RNA extraction from different apple tissues rich in polyphenols and polysaccharides for cDNA library construction. Plant Mol Bio Rep 2004, 50:859-865.

62. Ochman H, Gerber AS, Hartl DL: Genetic applications of an inverse polymerase chain reaction. Genetics 1988, 120:621-623.

63. Katoh K, Kuma K, Toh H, Miyata T: MAFFT version 5: improvement in accuracy of multiple sequence alignment. Nucleic Acids Res 2005, 33:511-518.

64. Maddison WP, Maddison DR: Mesquite: a modular system for evolutionary analysis. 2009 [http://mesquiteproject.org], Version 2.6.
65. Talavera G, Castresana J: Improvement of phylogenies after removing divergent and ambiguously aligned blocks from protein sequence alignments. Syst Biol 2007, 56:564-577.

66. Abascal F, Zardoya R, Posada D: ProtTest: selection of best-fit models of protein evolution. Bioinformatics 2005, 21:2104-2105.

67. Stamatakis A: RAxML-VI-HPC: Maximum Likelihood-based Phylogenetic Analyses with Thousands of Taxa and Mixed Models. Bioinformatics 2006, 22:2688-2690.

68. Stamatakis A, Hoover P, Rougemont J: A fast bootstrapping algorithm for the RAxML web-servers. Syst Biol 2008, 57:758-771.

69. The CIPRES Portals. CIPRES. [http://www.phylo.org/sub_sections/portal].

70. FigTree version 1.3.1. Distributed by the authors. [http://tree.bio.ed.ac.uk].

71. Stevens PF: Angiosperm phylogeny website. 2010 [http://www.mobot.org/ MOBOT/research/APweb/, 2001 onwards. Version 11.

doi:10.1186/2041-9139-2-14

Cite this article as: Abercrombie et al:: Developmental evolution of flowering plant pollen tube cell walls: callose synthase (CalS) gene expression patterns. EvoDevo 2011 2:14

\section{Submit your next manuscript to BioMed Central and take full advantage of:}

- Convenient online submission

- Thorough peer review

- No space constraints or color figure charges

- Immediate publication on acceptance

- Inclusion in PubMed, CAS, Scopus and Google Scholar

- Research which is freely available for redistribution

Submit your manuscript at www.biomedcentral.com/submit
C) Biomed Central 